\title{
sciendo
}

Int. J. of Applied Mechanics and Engineering, 2020, vol.25, No.1, pp.75-91

DOI: $10.2478 /$ ijame-2020-0006

\section{THE COMPLEX GINZBURG LANDAU MODEL FOR AN OSCILLATORY CONVECTION IN A ROTATING FLUID LAYER}

\author{
S.H. MANJULA \\ Department of Mathematics, VFSTR, Guntur \\ Vadlamudi-522213, Andhra Pradesh, INDIA \\ P. KIRAN* and P. RAJ REDDY \\ Department of Mathematics \\ Chaitanya Bharathi Institute of Technology \\ Gandipet, Hyderabad, Telangana-500075, INDIA \\ E-mail: pallekiran_maths@cbit.ac.in \\ B.S. BHADAURIA \\ Department of Applied Mathematics \\ Babasaheb Bhimrao Ambedkar University \\ VidyaVihar, Raebareli Road, Lucknow - 226025, INDIA
}

\begin{abstract}
A weakly nonlinear thermal instability is investigated under rotation speed modulation. Using the perturbation analysis, a nonlinear physical model is simplified to determine the convective amplitude for oscillatory mode. A non-autonomous complex Ginzburg-Landau equation for the finite amplitude of convection is derived based on a small perturbed parameter. The effect of rotation is found either to stabilize or destabilize the system. The Nusselt number is obtained numerically to present the results of heat transfer. It is found that modulation has a significant effect on heat transport for lower values of $\omega_{f}$ while no effect for higher values. It is also found that modulation can be used alternately to control the heat transfer in the system. Further, oscillatory mode enhances heat transfer rather than stationary mode.
\end{abstract}

Key words: convection, finite amplitude, nonlinear theory, rotation modulation, Nusselt number.

\section{Introduction}

The interest in the phenomenon of natural convection under externally modulated hydrodynamic configuration has been growing in engineering and thermal sciences in both theoretical and experimental studies. An excellent review on thermal convection was written by Chandrasekhar [1] and Drazin and Reid [2]. It is required to consider external phenomena, such as rotation, double diffusive convection, magnetic field, viscoelastic fluids, etc., for existing oscillatory mode of convection. In the present article, we consider rotation, and study Rayleigh-Bénard problem in a rotating fluid layer under rotation speed modulation. Donnely [3] was the first to study thermal instability in a rotating fluid layer under rotation speed modulation using perturbation method for free-free boundaries. For free-free boundaries conditions the normal velocity is zero and the tangential stress is zero at the wall. While calculating the shift in the critical Rayleigh number, he found that the system can be destabilized or stabilized by suitably tuning the frequency of rotation speed modulation. The rotation speed modulation was the originating idea of thermal (Venezian [4]) and gravity (Gresho and Sani [5]) modulations.

\footnotetext{
* To whom correspondence should be addressed
} 
The effect of temperature modulation and the effect of modulation of rotation speed in the TaylorCouette instability have been investigated in detail both theoretically and experimentally by Ahlers et al. [6], Niemela and Donnelly [7], Walsh and Donnelly [8]. The studies related to thermal modulation are described in [9], [10], [11]. The effect of through flow and thermal modulation on a viscoelastic fluid saturated porous medium is investigated by Kiran et al. [9]. A weak nonlinear analysis is used to study the oscillatory mode of convection using Ginzburg Landau model. It is found that heat transport can be controlled effectively by a mechanism that is external to the system. Bhattacharjee [12] studied the effect of a rotation speed modulation of rotating fluid layer and he found that the threshold of convection can be raised or lowered depending on the Prandtl number and rotation speed. Om et al. [13] investigated the effect of rotation speed modulation on the onset of free convection in a rotating porous layer placed away from the axis of rotation. They found that for suitable combinations of values of frequency and amplitude of modulation, it is possible to delay or advance the onset of centrifugal convection. Om et al. [14] investigated a non-uniform rotation speed on the onset of free convection in a rotating porous layer about the $z$-axis. They found that the modulated rotation speed has a stabilizing effect on the onset of convection for different values of modulation frequency and the other physical parameters involved. Bhadauria and Kiran [15] performed a weakly nonlinear thermal instability in a rotating fluid layer under rotation speed modulation using the Landau model. They found that instabilities are dominant for low frequencies and for small amplitudes. The effect of gravity modulation on an oscillatory convection in a rotating fluid layer is investigated by Kiran et al. [16].

There are few studies on thermal convection for rotational effects (Rauscher and Kelly [17], Malashetty and Swamy [18] and Bhadauria et al. [19]) either for linear and weakly nonlinear stationary theories. Thus, it is clear that, not even a single study is reported on weakly nonlinear oscillatory mode of convection under rotation speed modulation. Bhadauria and Kiran [20], [21] are the first who investigated a weakly nonlinear oscillatory convection for both temperature and gravity modulations. Using a complex Ginzburg-Landau equation (involving complex coefficients) they found better results than stationary convection. Thermal convection in a rotating fluid layer under the effect of temperature modulation is investigated by Kiran and Bhadauria [22]. The complex Ginzburg-Landau model was applied to derive amplitude of oscillatory convection. With the same configuration for porous media thermal and gravity modulations are developed by Kiran et al. [23], [24]. Their studies show that heat transfer results are enhanced by oscillatory case and controlled by modulation. Recently, Kiran and Narasimhulu [25] investigated the same problem for porous media saturated with a nanofluid. They used the Fourier series model for finite amplitude convection and determined heat transfer results under rotational speed modulation. Kiran et al. [26] investigated weakly nonlinear thermal convection using the complex Ginzburg Landau model under magnetic field modulation. The literature shows that no model is explained with nonlinear thermal oscillatory convection under the effects of time dependent rotational effects.

The study of weakly nonlinear oscillatory convection in a non-Newtonian fluid saturating a porous medium under temperature and gravity modulation is given in [27, 29]. Using the complex Ginzburg Landau equation they studied finite amplitude heat and mass transfer [30]. The study of weak nonlinear analysis of magneto-convection under magnetic field modulation is given by Bhadaria and Kiran [31]. Thermal instability in a rotating media is presented in the studies of [32-34]. The effect of gravity modulation on thermal instability in a fluid saturation porous media is given by Siddheshwar et al. [35], Bhadaria et al. [36] and Kiran [37, 38]. They investigated g-jitter effect on chaotic convection and binary convection. They found that g-jitter effects are found to stabilize the system and delay the chaos in the system. The study of the combined effect of internal heating and time periodic gravity modulation on thermal instability in a viscoelastic fluid layer is given by Bhadauria et al. [39]. Using a complex non-autonomous Ginzburg-Landau equation weak non-linear stability analysis was performed. It is found that overstability advances the onset of convection more with internal heating, hence increases heat transfer. It is also found that modulation has a destabilizing effect at low frequencies and a stabilizing effect at high frequencies. The effect of sinusoidal concentration modulation on thermal convection is investigated by Kiran [40]. The finite amplitude analysis is given by using the Landau equation.

For a stationary case, the effect of rotation speed modulation is investigated by Bhadauria and Kiran [15]. They investigated the sensitivity of viscosity and internal heating on heat transfer under rotational speed 
modulation. The weak nonlinear study was employed using the stationary Ginzburg Landau equation under thermal modulation. Recently, Kiran et al. [22], investigated an oscillatory convection under rotational effect. While deriving the complex Ginzburg-Landau equation finite amplitude convection is discussed. They found the condition for the existence of the model on $\operatorname{Pr}<1$, and other constraints to oscillatory critical Rayleigh number.

No data are reported on the study of rotational speed modulation on thermal convection for oscillatory mode. This paper is aimed to study heat transfer results in a rotating fluid layer while considering rotation speed modulation for an oscillatory mode of convection. Using the complex Ginzburg Landau model an oscillatory mode of thermal instability is demonstrated under rotational speed modulation.

\section{Mathematical formulation}

Consider an incompressible horizontal viscous fluid layer of thickness $d$, confined between two parallel plates at $z=0$ and $z=d$. The layer is heated from below and cooled from above. The Cartesian frame of reference is considered in such a way that the origin lies on the lower plane and the direction of the $z$-axis is vertical upward.

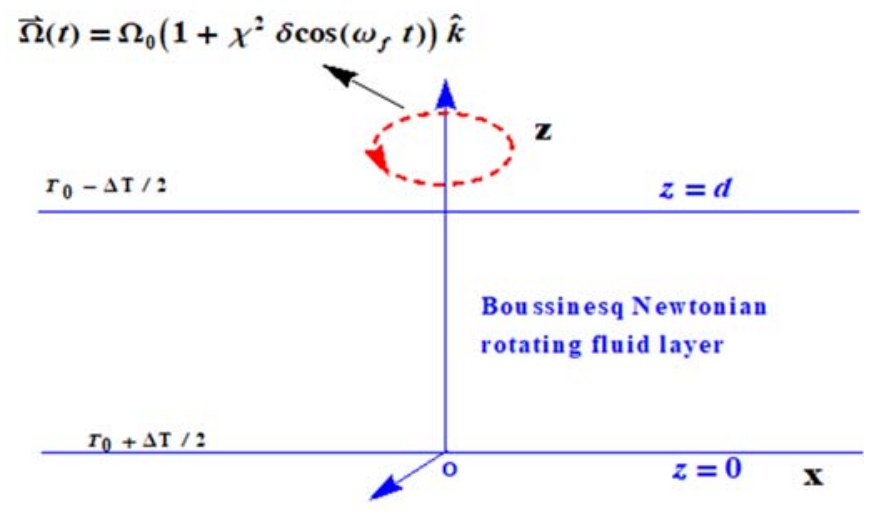

Fig.1. Physical representation of the problem.

The fluid layer is rotating with variable rotation speed $\Omega=(0,0, \Omega(t))$ about its $z$-axis. The fluid layer is of Boussinesq approximation where the density variations in the system are sufficiently small to be neglected, except where they appear in terms multiplied by gravity. Under these assumptions, the governing equations of the problem are given by Chandrasekhar [1]

$$
\begin{aligned}
& \Delta \cdot \boldsymbol{q}=0, \\
& \frac{\partial \boldsymbol{q}}{\partial t}+(\boldsymbol{q} \cdot \nabla) \boldsymbol{q}+(2 \boldsymbol{\Omega} \times \boldsymbol{q})=-\frac{1}{\rho_{0}} \nabla_{p}+\frac{\rho}{\rho_{0}} \boldsymbol{g}+u \nabla^{2} \boldsymbol{q}, \\
& \frac{\partial T}{\partial t}+(\boldsymbol{q} \cdot \Delta) T=\mathrm{K}_{T} \Delta^{2} T, \\
& \rho=\rho_{0}\left[1-\alpha_{T}\left(T-T_{0}\right)\right],
\end{aligned}
$$

where $\boldsymbol{q}=(u, v, w)$ is the fluid velocity, $T$ is temperature, $p$ is the reduced pressure, $\boldsymbol{\kappa}_{T}$ is the thermal diffusivity tensor, $\alpha_{T}$ is the thermal expansion coefficient, $\mu$ is the dynamic viscosity of the fluid, $v$ is the 
kinematic viscosity, $\rho$ is the density, $\rho_{0}$ and $T_{0}$ are the reference density and temperature, respectively, $\boldsymbol{g}$ is the gravity field $(0,0,-g)$ and $t$ is the time. The considered rotation speed vector (angular velocity vector $(0,0, \Omega(t))$ ) which is varying sinusoidal with time, is defined as (given by Om et al. [14] and Bhadauria et al. [19])

$$
\Omega(t)=\Omega_{0}\left(1+\chi^{2} \delta \cos \left(\omega_{f} t\right)\right) \hat{k}
$$

where $\delta$ is the small amplitude of modulation, $\omega_{f}$ is the modulation frequency. The equations are then non-dimensionalized using the physical variables: $(x, y, z)=d\left(x^{*}, y^{*}, z^{*}\right), t=\frac{d^{2}}{\kappa_{T}} t^{*}, \boldsymbol{q}=\frac{\kappa_{T}}{d} \boldsymbol{q}^{*}, \psi=\kappa_{T} \psi^{*}$ and $T=\Delta T T^{*}$ while eliminating the pressure term, introducing the stream function $\psi$ we obtain the following dimensionless governing system (Bhadauria et al. $[15,19]$ )

$$
\begin{aligned}
& \left(\frac{1}{\operatorname{Pr}} \frac{\partial}{\partial t} \nabla^{2}-\nabla^{4}\right) \psi+\operatorname{Ra} \frac{\partial T}{\partial x}-\sqrt{\operatorname{Ta}}\left(1+\chi^{2} \delta \cos \left(\omega_{f} t\right) \frac{\partial V}{\partial z}=\frac{1}{\operatorname{Pr}} \frac{\partial\left(\psi, \nabla^{2} \psi\right)}{\partial(x, z)},\right. \\
& -\frac{d T_{b}}{d z} \frac{\partial \psi}{\partial x}+\left(\frac{\partial}{\partial t}-\nabla^{2}\right) T=\frac{\partial(\psi, T)}{\partial(x, z)}, \\
& \sqrt{\operatorname{Ta}}\left(1+\chi^{2} \delta \cos \left(\omega_{f} t\right) \frac{\partial \psi}{\partial z}+\left(\frac{1}{\operatorname{Pr}} \frac{\partial}{\partial t}-\nabla^{2}\right) V=\frac{1}{\operatorname{Pr}} \frac{\partial(\psi, V)}{\partial(x, z)} .\right.
\end{aligned}
$$

The definition of dimensionless numbers: Prandtl number $\operatorname{Pr}=\frac{v}{\kappa T}$, thermal Rayleigh number $\mathrm{Ra}=\frac{\alpha_{T} g \Delta T d^{3}}{v k_{T}}$ and Taylor number $\mathrm{Ta}=\left(\frac{2 \Omega d^{2}}{v}\right)^{2}$. The stream function representation used in Eqs (2.6) (2.8) is based on Bhadauria et al. $[15,19]$. The disturbances are accounted here in the $x$ and $z$ directions. It is not that the perturbations in the $y$-direction can not be neglected, in general they cannot, but there is a plane perpendicular to the $x-y$ plane and forming an angle $\tan ^{-1}\left(\frac{v}{u}\right)$ with $x$-direction that depends on $\Omega$ where the stream function exists. On this tilted plane there is no velocity component normal to the plane, and therefore this is regarded as the oblique plane containing the streamlines [see Chandrasekhar [1] (page 109 for a graphical description of the convection pattern in the oblique plane) and Veronis [34]]. The wavenumber in the oblique plane containing streamlines can be described in the form $a_{c}=\frac{\pi}{(1+\mathrm{Ta})^{1 / 4}}$. This shows that the wavelength of the roll measured in the plane containing the streamlines is not independent of rotation (i.e. it is a function of the Taylor number), in contrast to the corresponding problem in pure fluids, where the wavelength in the oblique plane containing the streamlines was found to be identical to the value obtained for convection in absence of rotation (Veronis [34]). The above system of Eqs (2.6)-(2.8) will be solved by considering stress free and isothermal boundary conditions $\psi=\frac{\partial^{2} \psi}{\partial z^{2}}=\frac{\partial V}{\partial z}=T=0$ on $z=0, z=1$. 
Introducing a small perturbation parameter $\chi$ that shows deviation from the critical state of onset of convection, the variables for a weak nonlinear state may be expanded as a power series in terms of $\chi$ (as in Venezian [4], Bhadauria and Kiran [27, 28, 36-39] and Malkus and Veronis [30] to consider effects of finiteamplitude convection)

$$
\begin{aligned}
& \mathrm{Ra}=R_{0}+\chi R_{1}+\chi^{2} R_{2}+\chi^{3} R_{3}+\ldots, \\
& \psi=\chi \psi_{1}+\chi^{2} \psi_{2}+\chi^{3} \psi_{3}+\ldots \\
& T=\chi T_{1}+\chi^{2} T_{2}+\chi^{3} T_{3}+\ldots \\
& V=\chi V_{1}+\chi^{2} V_{2}+\chi^{3} V_{3}+\ldots
\end{aligned}
$$

where $R_{0}$ is the critical Rayleigh number at which the onset of convection takes place in the absence of temperature modulation. By using the definition $\chi$ the Rayleigh number may be expanded as $R a=R_{0}\left(1+\chi^{2}\right)$, where $R_{0}$ is the critical Rayleigh number for a layer heated from below and subjected to modulation.

\section{Bifurcation of periodic solution}

For anticipated frequency shift along the bifurcation solution (Kim et al. [27]), we introduce the fast time scale of time $\tau$ and the slow time scale of $s$. Therefore, the scaling of time variable is such that $\frac{\partial}{\partial t}=\frac{\partial}{\partial \tau}+\chi^{2} \frac{\partial}{\partial s}$. In the first order problem, the nonlinear term in energy equation will vanish. Therefore, the first order problem reduces to the linear stability problem for oscillatory mode of convection.

\subsection{Lowest order system}

In this order we obtain the following system of matrix (which is similar to the linear theory problem)

$$
\left.\left(\begin{array}{ccc}
\left(\frac{1}{\operatorname{Pr}} \frac{\partial}{\partial r} \nabla^{2}-\nabla^{4}\right) & R_{0} \frac{\partial}{\partial x} & -\sqrt{\mathrm{Ta}} \frac{\partial}{\partial z} \\
\frac{\partial}{\partial x} & \frac{\partial}{\partial r}-\nabla^{2} & 0 \\
\sqrt{\operatorname{Ta}} \frac{\partial}{\partial z} & 0 & \left(\frac{1}{\operatorname{Pr}} \frac{\partial}{\partial r}-\nabla^{2}\right.
\end{array}\right)\right)\left[\begin{array}{l}
\psi_{1} \\
T_{1} \\
V_{1}
\end{array}\right]=\left[\begin{array}{l}
0 \\
0 \\
0
\end{array}\right] .
$$

The solution of the lowest order system subject to the boundary conditions given in Eq.(2.9), is chosen as in Kim et al. [27], see also [27, 28, 36, 37]

$$
\begin{aligned}
& \psi_{1}=\left(\mathrm{A}_{l}(s) e^{i \omega T}+\overline{\mathrm{A}}_{1}(s) e^{-i \omega T}\right) \sin a x \sin \pi z, \\
& T_{1}=\left(B_{1}(s) e^{i \omega T}+\bar{B}_{1}(s) e^{-i \omega T}\right) \cos a x \sin \pi z,
\end{aligned}
$$




$$
V_{l}=\left(\mathbb{C}_{l}(s) e^{i \omega T}+\bar{B}_{l}(s) e^{-i \omega T}\right) \sin a x \cos \pi z
$$

The undetermined amplitudes in Eqs (2.2)-(3.4) are functions of slow time scale and are related by the following relation

$$
\begin{aligned}
& B(s)=-\frac{a}{c+i \omega} \mathrm{A}(s), \\
& \mathbb{C}(s)=-\frac{\pi \sqrt{\mathrm{Ta}}}{\left(c+\frac{i \omega}{\mathrm{Pr}}\right)} \mathrm{A}(s)
\end{aligned}
$$

where $c=a^{2}+\pi^{2}$. The values of the critical Rayleigh number and the corresponding frequency of periodic convection are

$$
\begin{aligned}
& R_{0}=\frac{c^{3}-\frac{c \omega^{2}}{\operatorname{Pr}}}{a^{2}}+\frac{\pi^{2} \mathrm{Ta}}{a^{2}}\left(\frac{c^{2}+\frac{\omega^{2}}{\operatorname{Pr}}}{c^{2}+\frac{\omega^{2}}{\operatorname{Pr}^{2}}}\right), \\
& \omega^{2}=\operatorname{Pr}^{2}\left(\frac{\pi^{2} \operatorname{Ta}(1-\operatorname{Pr})}{c(1+\operatorname{Pr})}-c^{2}\right) .
\end{aligned}
$$

It is obvious from the above expression Eq.(3.11) that $\omega$ becomes a complex quantity when Pr exceeds one, since $\omega$ must be a positive real quantity (for the oscillatory convection to be possible), Pr values are considered less than one. Therefore, for pure fluids, overstability is possible only for values of the Prandtl numbers less than 1 to obtain real frequencies. For stationary mode of convection, we obtain the critical Rayleigh number when $(\omega=0)$

$$
R_{0}=\frac{c^{3}+\pi^{2} \mathrm{Ta}}{a^{2}}
$$

which is the result obtained by Bhadauria et al. [17]. It can be observed that the mode of oscillatory convection exists when the Taylor number satisfies the following inequality

$$
\operatorname{Ta}>\frac{(1+\operatorname{Pr})}{\pi^{2}(1-\operatorname{Pr})} c^{3}
$$

The wave number corresponding to the Rayleigh numbers can be obtained by minimizing the Rayleigh number with respect to the critical wave number.

\subsection{Second order} equation

At this state the nonlinear effects are accounted in terms of the Jacobian. It is given by the following

$$
\frac{\partial\left(\psi_{1}, T_{1}\right)}{\partial(x, z)}=\frac{\pi a}{2}\left\{\mathrm{~A}(s) B(s) e^{2 i \omega T}+\overline{\mathrm{A}}(s) \bar{B}(s) e^{-2 i \omega T}+\overline{\mathrm{A}}(s) \bar{B}(s)+\overline{\mathrm{A}}(s) \bar{B}(s)\right\} \sin 2 \pi \mathrm{z} .
$$


The solution of the above equation depends on the first order solutions. From the above relation, we can deduce that the velocity and temperature fields have the terms having frequency $2 \omega$ and independent of past time scale. Thus, we write the second order temperature term as follows

$$
T_{2}=\left\{T_{20}+T_{22} e^{2 i \omega \tau}+T_{22} e^{-2 i \omega \tau}\right\} \sin 2 \pi z
$$

where $T_{22}$ and $T_{20}$ are temperature fields with the terms having the frequency $2 \omega$ and independent of fast time scale, respectively. The solutions of the second order problem are

$$
\begin{aligned}
& \psi_{2}=0, \\
& T_{20}=\frac{a}{8 \pi}\left\{\mathrm{A}_{1}(s) B_{1}(s) \mathrm{A}_{1}(s) B_{1}(s)\right\}, \\
& T_{22}=\frac{\pi a}{8 \pi^{2}+4 i \omega} \mathrm{A}_{1}(s) B_{1}(s) .
\end{aligned}
$$

For large-scale zonal velocity $V$, it is necessary to capture the shear that builds up across convection and enters at $O(\chi)$; its inclusion is a consequence of the phase-like quality of the variable $V$, i.e. the invariance of Eqs (2.1)-(2.4) with the boundary conditions Eq.(2.9). We consider Eq.(3.16) for solving the system.

$$
V_{2}=\left\{V_{20}+V_{22} e^{2 i \omega \tau}+\bar{V}_{22} e^{-2 i \omega \tau}\right\} \sin a x .
$$

The solutions of the above equation are obtained as

$$
\begin{aligned}
& V_{20}=\frac{a}{8 \pi}\{\mathrm{A}(s) \mathbb{C}(s)+\mathrm{A}(\mathrm{s}) \mathbb{C}(s)\}, \\
& V_{22}=\frac{\pi a}{8 a^{2}+\frac{2 i \omega}{\operatorname{Pr}}} \mathrm{A}(s) \mathbb{C}(s) .
\end{aligned}
$$

The horizontal Nusselt number $\mathrm{Nu}$ for an oscillatory mode of convection is defined by [19]-[28]

$$
\mathrm{Nu}=1+\frac{\left[\frac{a}{2 \pi} \int_{0}^{\frac{2 \pi}{a}}\left(\frac{\partial T_{2}}{\partial z}\right) d x\right]_{z=0}}{\left[\frac{a}{2 \pi} \int_{0}^{\frac{2 \pi}{a}}\left(\frac{\partial T_{b}}{\partial z}\right) d x\right]_{z=0}}
$$

Substituting the expression of $\boldsymbol{T}_{2}$, given in Eq.(3.12) and $T_{b}=1-z$ one can simplify Eq.(3.19) as 


$$
\mathrm{Nu}=1+a^{2}\left(\frac{c}{2\left(c^{2}+\omega^{2}\right)}+\frac{\pi^{2}}{2 \sqrt{\left(4 \pi^{4}+\omega^{2}\right)} \sqrt{\left(c^{2}+\omega^{2}\right)}}\right)\left|A_{1}(s)\right|^{2} .
$$

It is clear from the relation Eq.(2.5) that the rotation speed modulation is effective at the third order and affects $\mathrm{Nu}$ through $A(s)$, which is evaluated at the third order. We also calculate the horizontally averaged Nusselt number, for the oscillatory mode of convection for understanding the results better

$$
\mathrm{N} \overline{\mathrm{u}}=\frac{1}{2 \pi} \int_{0}^{2 \pi} \mathrm{Nu} d s
$$

\subsection{Third order}

At this order finding solutions is very difficult due to complexity of the resultant equations. For the third-order system, the following relations are obtained

$$
\left(\begin{array}{ccc}
\left(\frac{1}{\operatorname{Pr}} \frac{\partial}{\partial r} \nabla^{2}-\nabla^{4}\right) & R_{0} \frac{\partial}{\partial x} & -\sqrt{\mathrm{Ta}} \frac{\partial}{\partial z} \\
\frac{\partial}{\partial x} & \frac{\partial}{\partial r}-\nabla^{2} & 0 \\
\sqrt{\mathrm{Ta}} \frac{\partial}{\partial z} & 0 & \left(\frac{1}{\operatorname{Pr}} \frac{\partial}{\partial r}-\nabla^{2}\right)
\end{array}\right)\left[\begin{array}{l}
\Psi_{3} \\
T_{3} \\
V_{3}
\end{array}\right]=\left[\begin{array}{c}
R_{31} \\
R_{32} \\
R_{33}
\end{array}\right]
$$

The expressions for $R_{31}, R_{32}$ and $R_{33}$ given in Eq.(3.22) are

$$
\begin{aligned}
& R_{31}=-\frac{1}{\operatorname{Pr}} \frac{\partial \nabla^{2} \Psi_{1}}{\partial s}-R_{0} \frac{\partial T_{2}}{\partial x}-R_{2} \frac{\partial T_{1}}{\partial x}+\sqrt{\mathrm{Ta}} \delta \cos \left(\omega_{f} s\right) \frac{\partial V_{1}}{\partial z}, \\
& R_{32}=\frac{\partial T_{1}}{\partial s}+\frac{\partial \psi_{1}}{\partial x}\left(\frac{\partial T_{2}}{\partial z}\right), \\
& R_{33}=-\frac{1}{\operatorname{Pr}} \frac{\partial V_{1}}{\partial s}-\frac{1}{\operatorname{Pr}} \frac{\partial \psi_{1}}{\partial z} \frac{\partial V_{2}}{\partial s}-\sqrt{\mathrm{Ta}} \delta \cos \left(\omega_{f} s\right) \frac{\partial \psi_{1}}{\partial z} .
\end{aligned}
$$

Using the expressions of $R_{31}, R_{32}$ and $R_{33}$ and applying the following solvability condition (Bhadauria and Kiran $[20,21])$ for the existence of a third order solution, we have

$$
\int_{0}^{\frac{2 \pi}{a_{c}}} \int_{0}^{1}\left(R_{31} \widehat{\psi}_{1}+R_{32} \widehat{T}_{1}+R_{33} \widehat{V}_{1}\right) d x d z=0
$$

Simplifying the above equation yields the complex Ginzburg-Landau equation for unknown complex amplitude $A(s)$ of the convection 


$$
\frac{\partial A_{l}(s)}{\partial s}-\Upsilon_{1}^{-1} F(s)+\Upsilon_{1}^{-1} k_{l}\left|A_{l}(s)\right|^{2} A_{l}(s)=0
$$

The coefficients given in Eq.(3.27) are defined by

$$
\begin{aligned}
& \Upsilon_{1}=\left[\frac{c}{\operatorname{Pr}^{2}}-\frac{a^{2} R_{0}}{(c+i \omega)^{2}}-\frac{\pi^{2} T a}{\operatorname{Pr}\left(c+\frac{i \omega}{\operatorname{Pr}}\right)^{2}}\right], \quad F(S)=\left[\frac{a^{2}}{(c+i \omega)}\left(R_{2}+R_{0} \delta_{2} \cos \left(\omega_{f} s\right)\right)\right], \\
& k_{l}=\left[\frac{a^{4} R_{0} c}{4\left(c^{2}+\omega^{2}\right)(c+i \omega)}+\frac{\pi^{2} a^{4} R_{0}}{\left(8 \pi^{2}+4 i \omega\right)(c+i \omega)^{2}}+\right. \\
& \left.+\frac{\pi^{4} a^{4} c T a}{4\left(c^{2}+\frac{\omega^{2}}{\operatorname{Pr}^{2}}\right)\left(c+\frac{i \omega}{\operatorname{Pr}}\right)}+\frac{\pi^{4} a^{2} T a}{2\left(4 a^{2}+\frac{i \omega}{\operatorname{Pr}}\right)\left(c+\frac{i \omega}{\operatorname{Pr}}\right)^{2}}\right]
\end{aligned}
$$

Writing $A_{l}(s)$ in the phase-amplitude form, we get $A_{l}(s)=\left|A_{l}(s)\right| e^{i \varphi}$ and substituting in Eq.(3.27), we get the following equation for the amplitude equations in terms of $\left|A_{I}(s)\right|$

$$
\begin{aligned}
& \frac{d\left|A_{l}(s)\right|^{2}}{d s}=2 p_{r}\left|A_{l}(s)\right|^{2}-2 l_{r}\left|A_{l}(s)\right|^{4}, \\
& \frac{d\left(p h\left(A_{l}(s)\right)\right)}{d s}=p_{i}-l_{i}\left|A_{l}(s)\right|^{2}
\end{aligned}
$$

where $\gamma_{1}^{-1} F(s)=p_{r}+i p_{i}, \gamma_{1}^{-1} k_{1}=l_{r}+i l_{i}$ and $p h($.$) represents the phase shift. We solve Eq.(3.29) using the inbuilt$ solution NDsolve of Mathematica 8, subjected to the condition $(0)=a_{0}$ where $a_{0}$ is chosen initial amplitude of convection. In our calculations we may use $R_{2}=R_{0}$ to keep the parameters to the minimum. The reader may also find an amplitude equation for stationary $(\omega=0)$ mode of convection, with time-periodic coefficients (Bhadauria et al. [15], [19] for the case of $R_{i}=V_{T}=0$.

\section{Results and discussions}

Since a comprehensive study of modulation (like thermal [4], [9], [10], [22], [23], [28], [29], gravity [5], [36], [37], [39] rotational [15], [16] and magnetic field [31] modulations) has been investigated while considering weak nonlinear stationary mode of convection. But, there is scarcity being observed (by [20], [21], [27], [28]) in the direction of non linear oscillatory convection. A close observation of modulation of Rayleigh-Bénard convection is to find external adjusting parameters which control the convective phenomenon in the system. It is also being identified by numerous authors; the rotating fluid layer reduces (stabilizing effect on the system) heat transfer drastically for the stationary mode of convection. Keeping this in mind, it is quite important to study thermal instability in a rotating fluid layer under oscillatory mode of convection. Hence, the present article is to track instability in a rotating fluid layer under time dependant rotation speed modulation. Following the earlier works of Bhadauria et al. [15], [19] and Malashetty and 
Swamy [18], the present article investigates a weakly nonlinear oscillatory mode of convection using the complex form of Ginzburg-Landau equation [27].

Bhadauria et al. [19], pointed that the oscillatory mode of convection exists for a certain range. [for a certain parameter range the overstability is a preferred mode [27] whereas for a stationary case no condition is required] of the system parameters where the Taylor number, Ta, and Prandtl number Pr, satisfy Eq.(3.10). With this relation, it is quite important to consider $\operatorname{Pr}<1$ for the present model, hence the effect of the local acceleration term affects the momentum equation. Vadász [32] pointed that only for low values of $\operatorname{Pr}$, oscillatory mode of convection is possible for a fluid layer, whereas for a porous medium, it is not limited to a particular domain of the Prandtl number. We consider low values of amplitude $\delta$ and frequency $\boldsymbol{\omega} \boldsymbol{f}$ of modulation, as at low values of modulation, heat transfer is maximum.

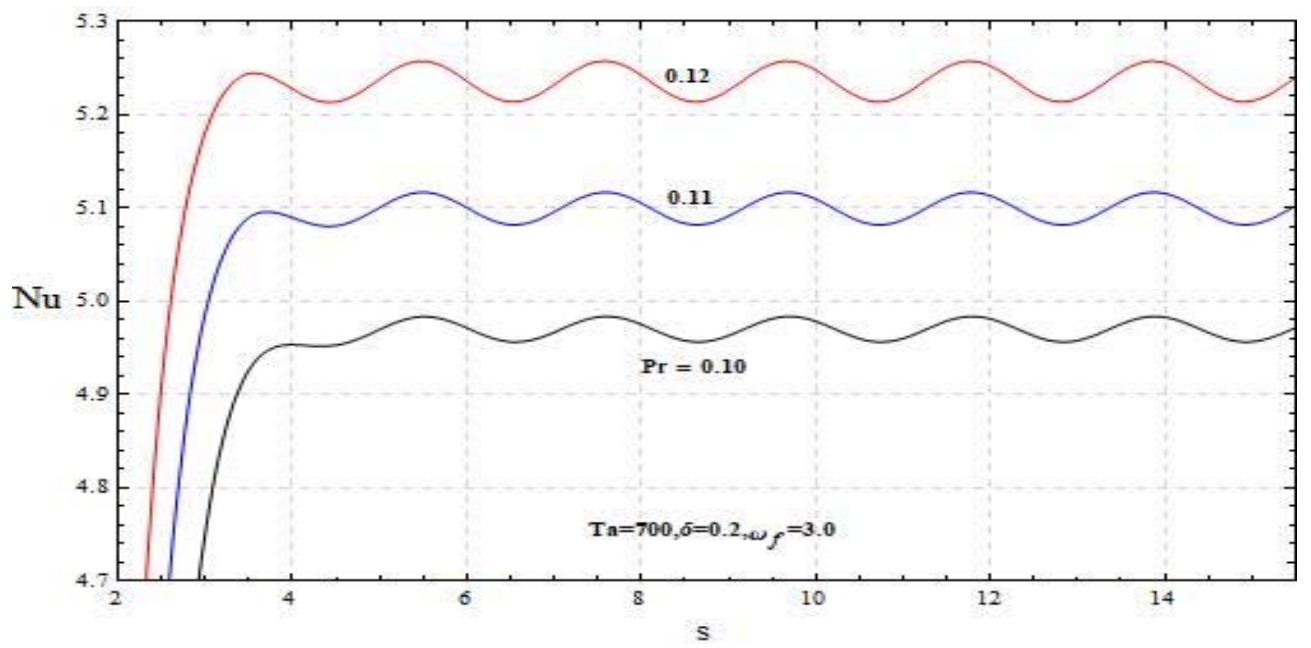

Fig.2. Effect of the Prandtl number on heat transfer.

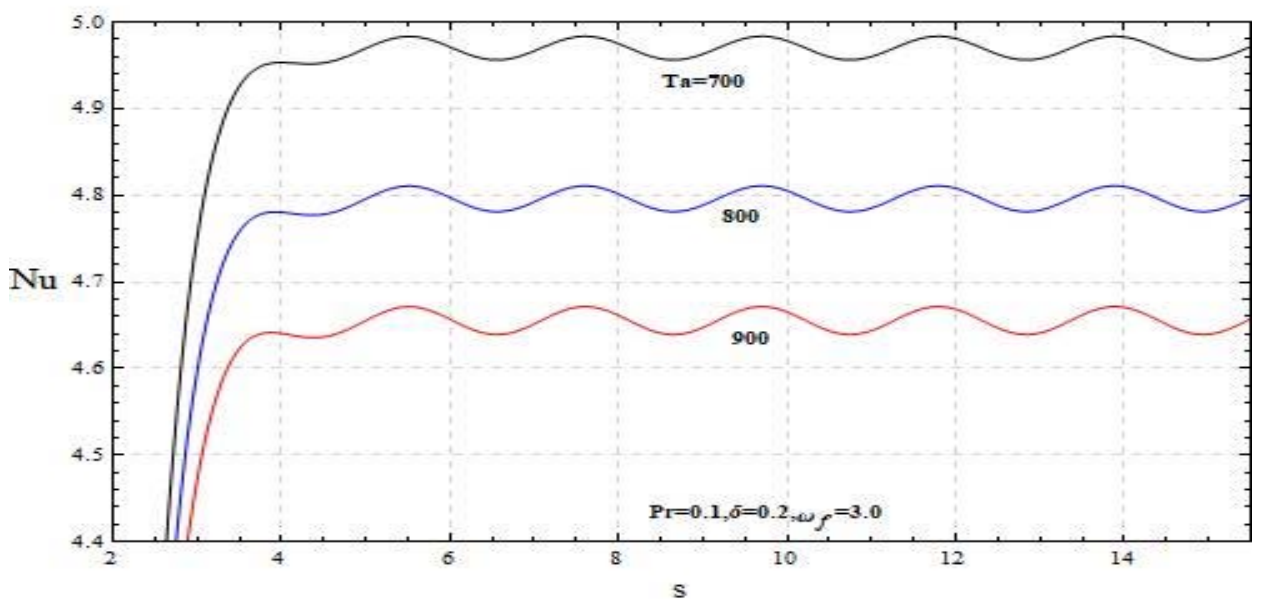

Fig.3. Effect of the Taylor number on heat transfer for the range $(\mathrm{Ta}<6000)$ where Ta has a stabilizing effect hence reduces heat transfer.

The results corresponding to modulation have been depicted in Figs 2-10, where we have drawn graphs for $\mathrm{Nu}$ versus slow time $s$. For lower values of time $s$, there is only a conduction state mass transfer 
showing $\mathrm{Nu}=1$. The Nusselt number increases with time $s$ and exceeds the value 1 , indicating that convective process occurs in the system.

Since Prandtl number takes values between 0 to 1 , thermal diffusivity dominates over momentum diffusivity. Especially, the Prandtl number controls the relative thickness of the momentum and thermal boundary layers. When $\operatorname{Pr}$ is small, it means that the heat diffuses quickly compared to the velocity. This means that for liquid metals the thickness of the thermal boundary layer is much bigger than the velocity boundary layer. When the Prandtl number increases (between 0 to 1 ), the critical value of the Rayleigh number decreases significantly, hence the Prandtl number has a tendency to destabilize the system.

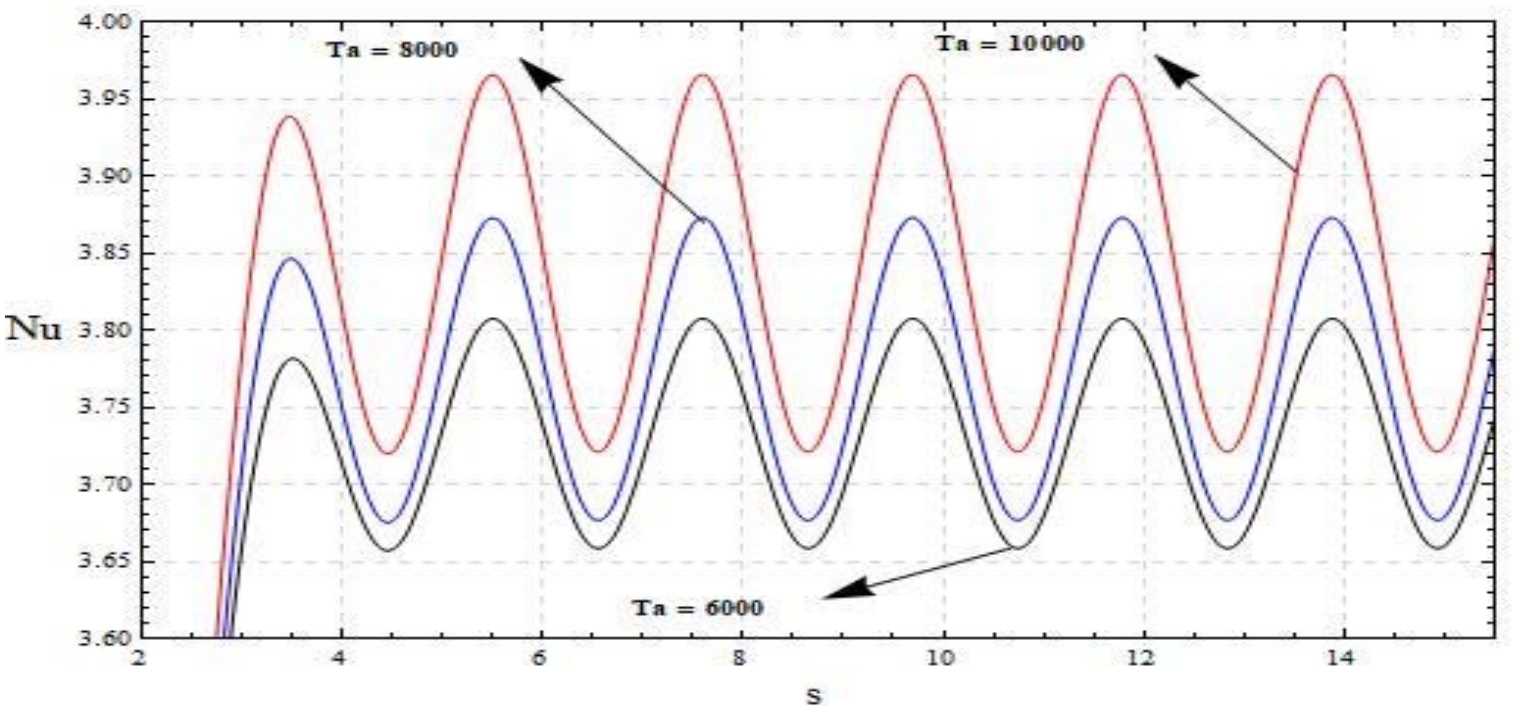

Fig.4. Effect of the Taylor number on heat transfer for the range $(6000<\mathrm{Ta})$ where Ta has a destabilizing effect, hence enhances heat transfer.

In Fig.2, upon increasing the value of Pr there is an increment in heat transfer. These results are compatible with the results obtained by Bhadauria et al. [15], [29] while considering low viscous fluids for the stationary and oscillatory mode of convection.

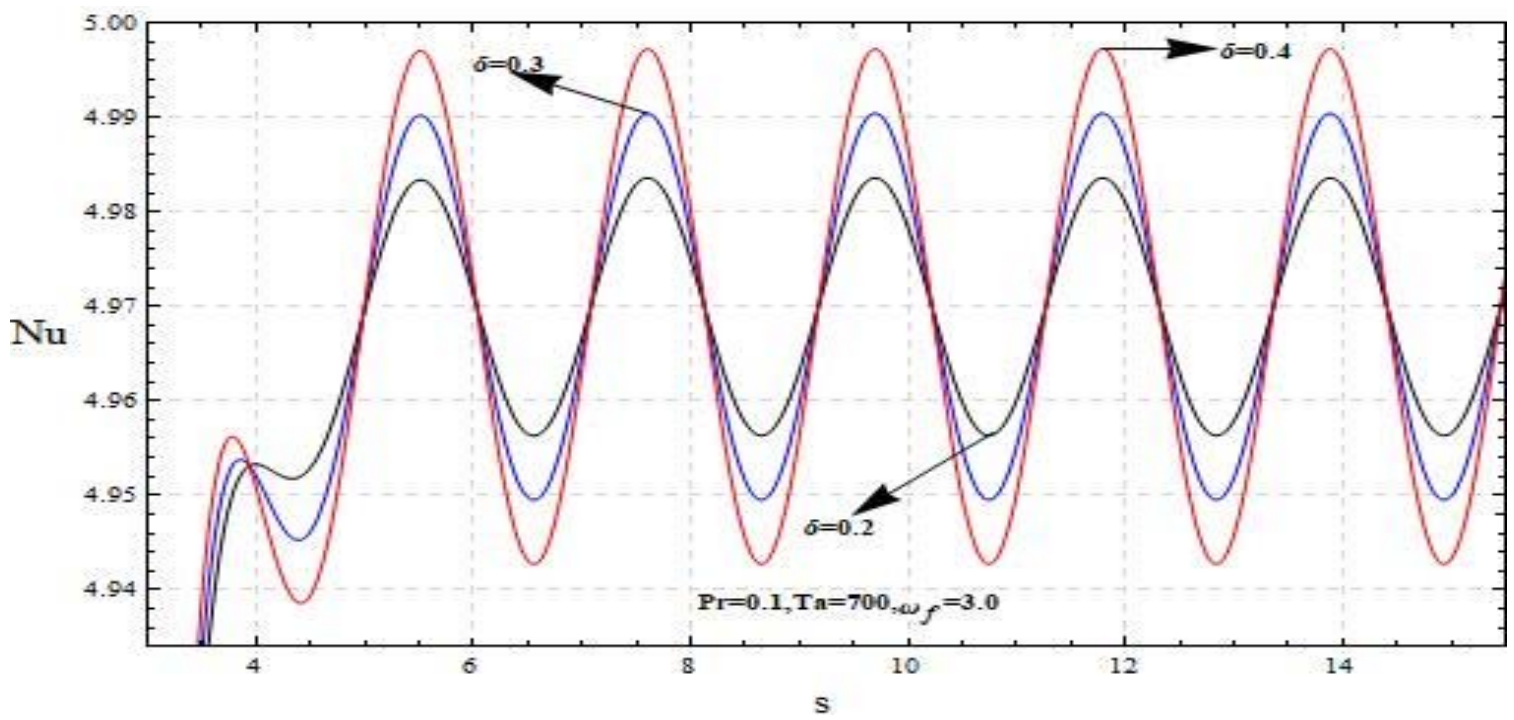

Fig.5. Effect of amplitude of modulation on heat transfer. 
In Fig. 3 the effect of the Taylor number reveals that Ta stabilizes the system for a certain finite range of the Taylor number (up to 6000 nearly). These results are quite natural and conform with the results of Bhadauria et al. [15], [19]. But, suddenly an opposite behavior may be noticed for large values of Ta $(\mathrm{Ta}>6000)$ presented in Fig.4. This behaviour confirms the results of Rauscher and Kelly [17]. In general or practically, considering higher values of $\mathrm{Ta}$ is not possible. It is also observed that Ta is multiple of modulation coefficient $\delta \cos (\omega f s)$ ) given in Eq.(3.27) in terms of $F(s)$.

As the Taylor number Ta varies more than 6000, heat transfer is greater and an amplitude of modulation also increases, these results conform with the results of Bhadauria and Kiran [15] and Kiran and Bhadauria [22].

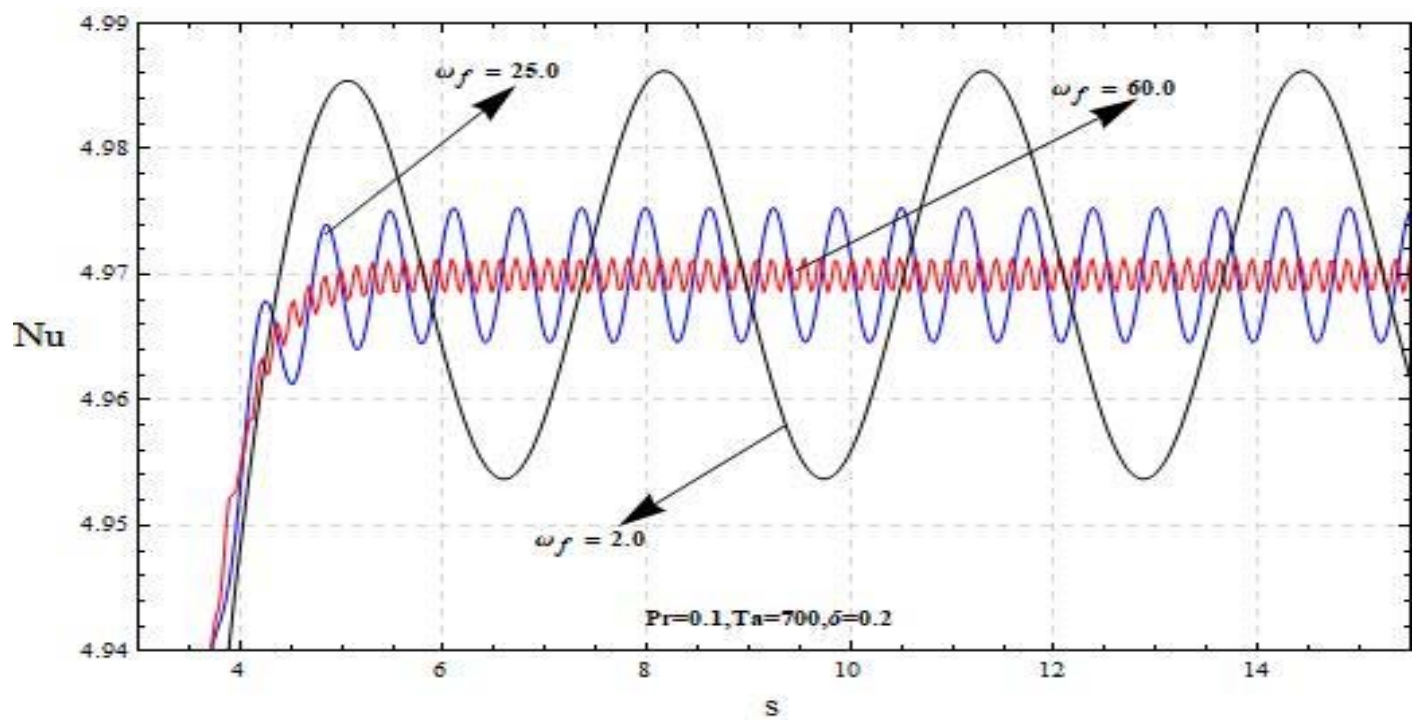

Fig.6. Effect of frequency of modulation on heat transfer.

The effect of amplitude $\delta$ and modulation frequency $\omega f$ on heat transfer is presented in Fig. 5 and 6. It is found that for certain ranges of frequencies the value of $\mathrm{Nu}$ is high, thus destabilizing the system, and low values stabilize the system. It is observed that the amplitude of modulation enhances heat transfer, but an opposite effect is observed for $\omega f$. From Fig.6, it is found that the effect of rotation speed modulation decreases as $\omega f$ increases; and, finally, when $\omega f$ is very large, the effect of modulation disappears altogether, thus confirming the results given in [4], [15]. A comparison of oscillatory and stationary mode of convection is presented in Fig.7. It is found that heat transfer is greater for the oscillatory mode of convection than for the stationary mode. The reason behind this is that for the oscillatory mode of convection, an additional quantity oscillatory frequency (the growth rate of disturbances) plays a critical role in the Rayleigh number and in the amplitude of convection. It is observed that

$$
\mathrm{Nu}^{\mathrm{st}}<\mathrm{Nu}^{\mathrm{OSC}}
$$

for the same wave number. This implies that oscillatory instabilities can set in before the stationary mode. Similar results have been obtained by Kim et al. [27], Bhadauria and Kiran [20], [21] and Kiran and Bhadauria [22]. Finally, for a better understanding of our results a comparison is made for different modulations. While adapting the works of Bhadauria and Kiran [20], [21], [35]-[38] for gravity modulation and Bhadauria and Kiran [28], [29] for thermal modulation, the comparison with rotation speed modulation is presented in Fig.8. It is observed that the results of gravity modulation coincide with the results of temperature modulation when the lower boundary is modulated. 


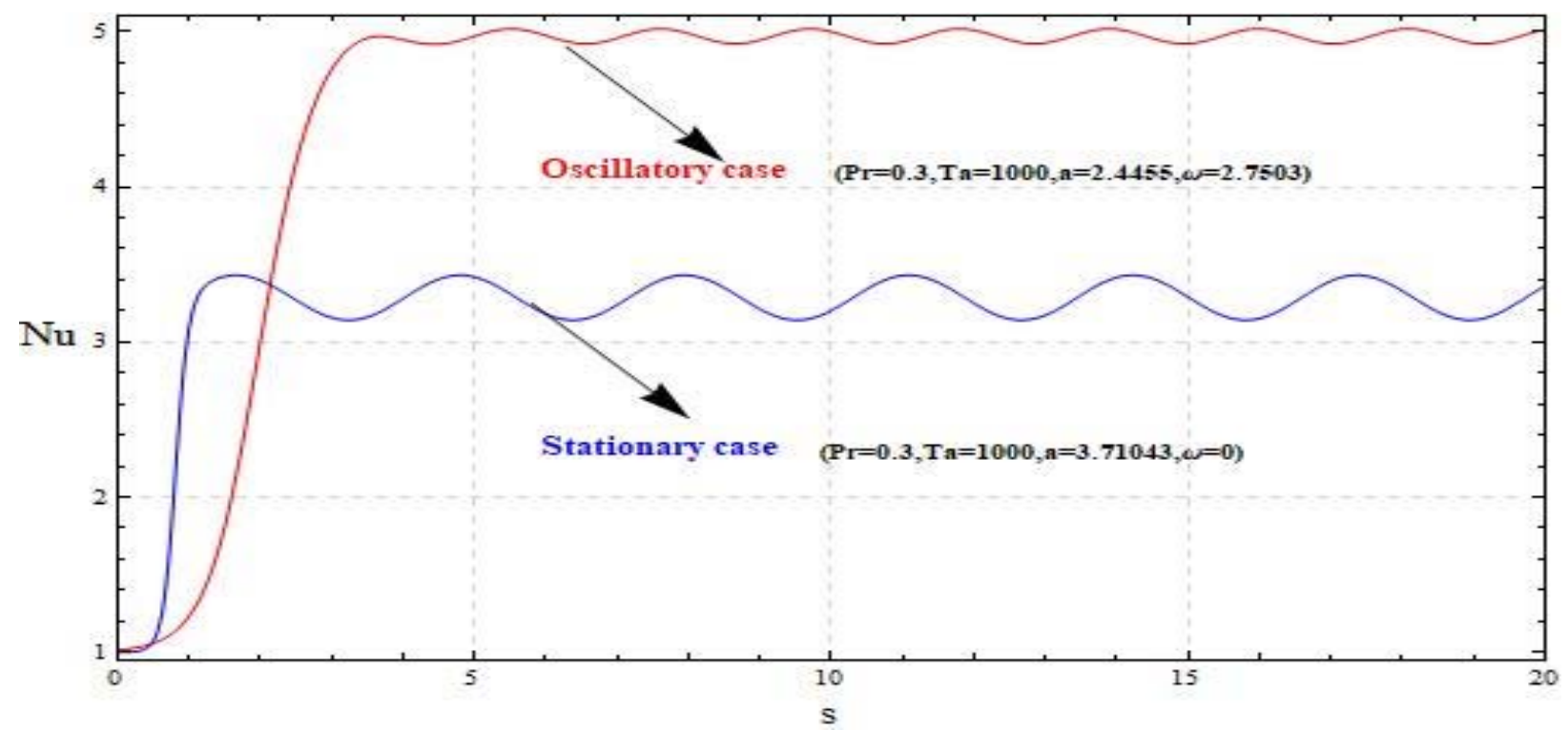

Fig.7. Comparison of stationary and oscillatory mode of convection.

But, for rotation speed modulation it is quite interesting to see that heat transfer is less for the current model than other modulations. This confirms that rotation speed modulation has a stabilizing effect and reduces heat transfer more than other two models. The results corresponding to modulated and un-modulated systems are presented in Fig.9.

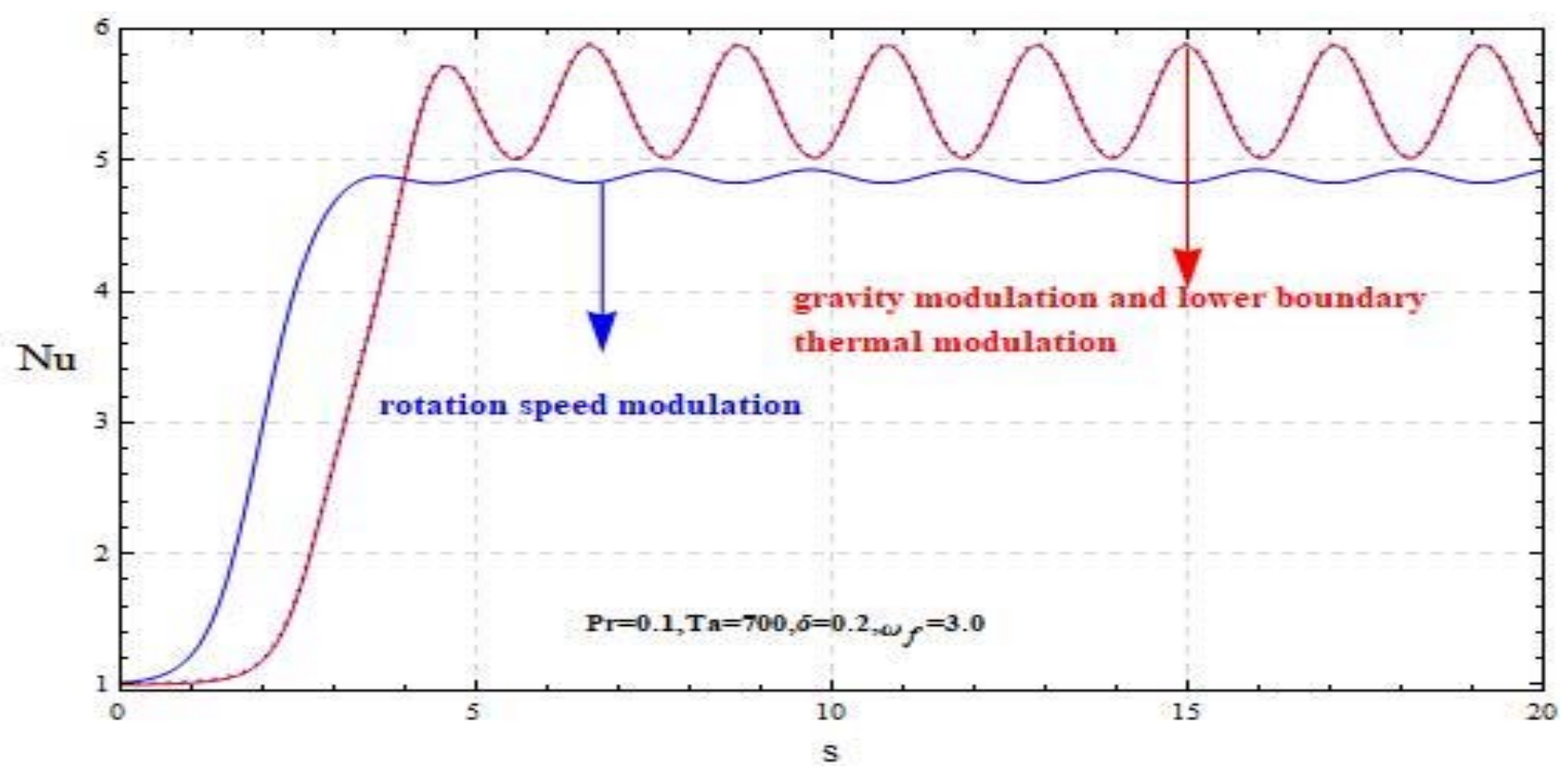

Fig.8. Comparison of various modulations.

For anun-modulated system the amplitude of modulation is obtained from Eq.(3.30), and no oscillations are found in Fig.9. This reveals that an unmodulated system do not alter heat transfer in the system. These results are similar to the case of in-phase modulation for thermal modulation $[28,29]$. 


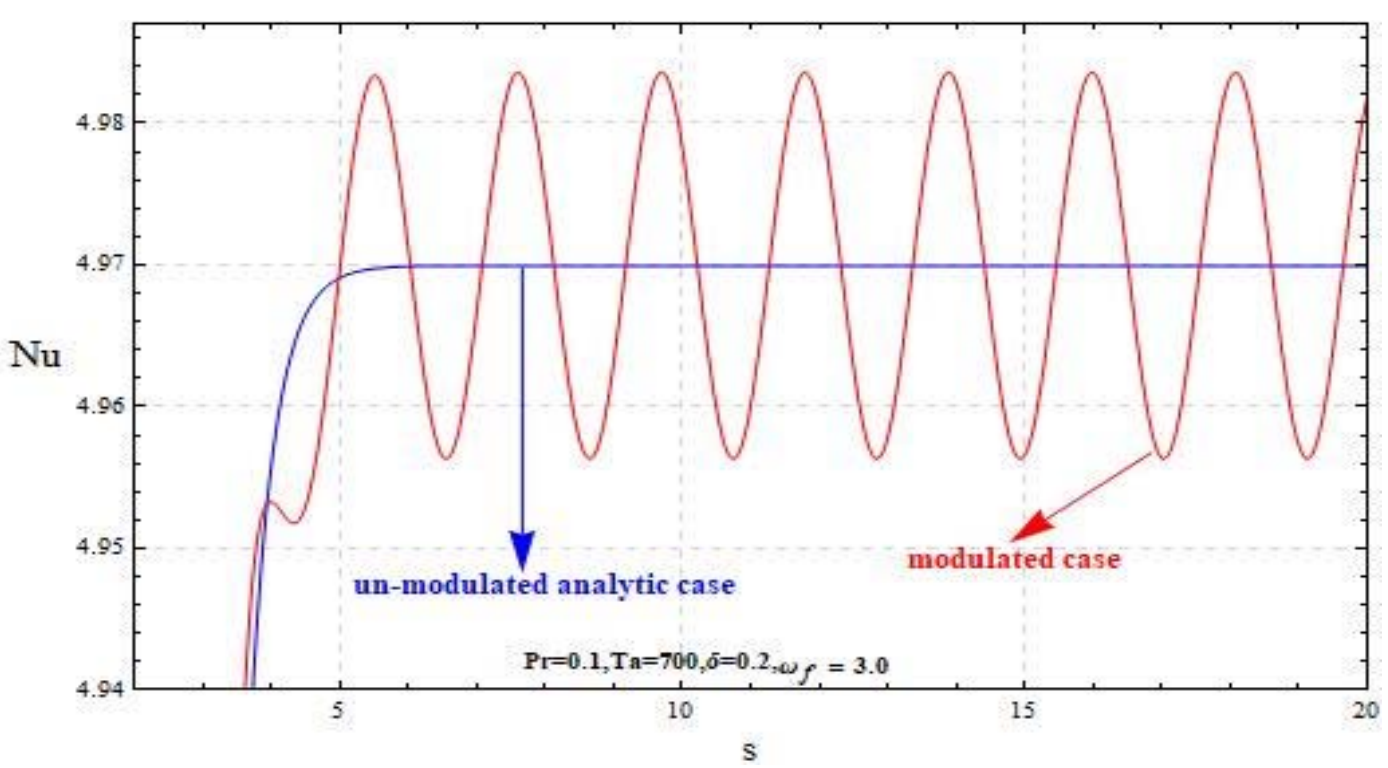

Fig.9. Comparison of modulated and un-modulated system.

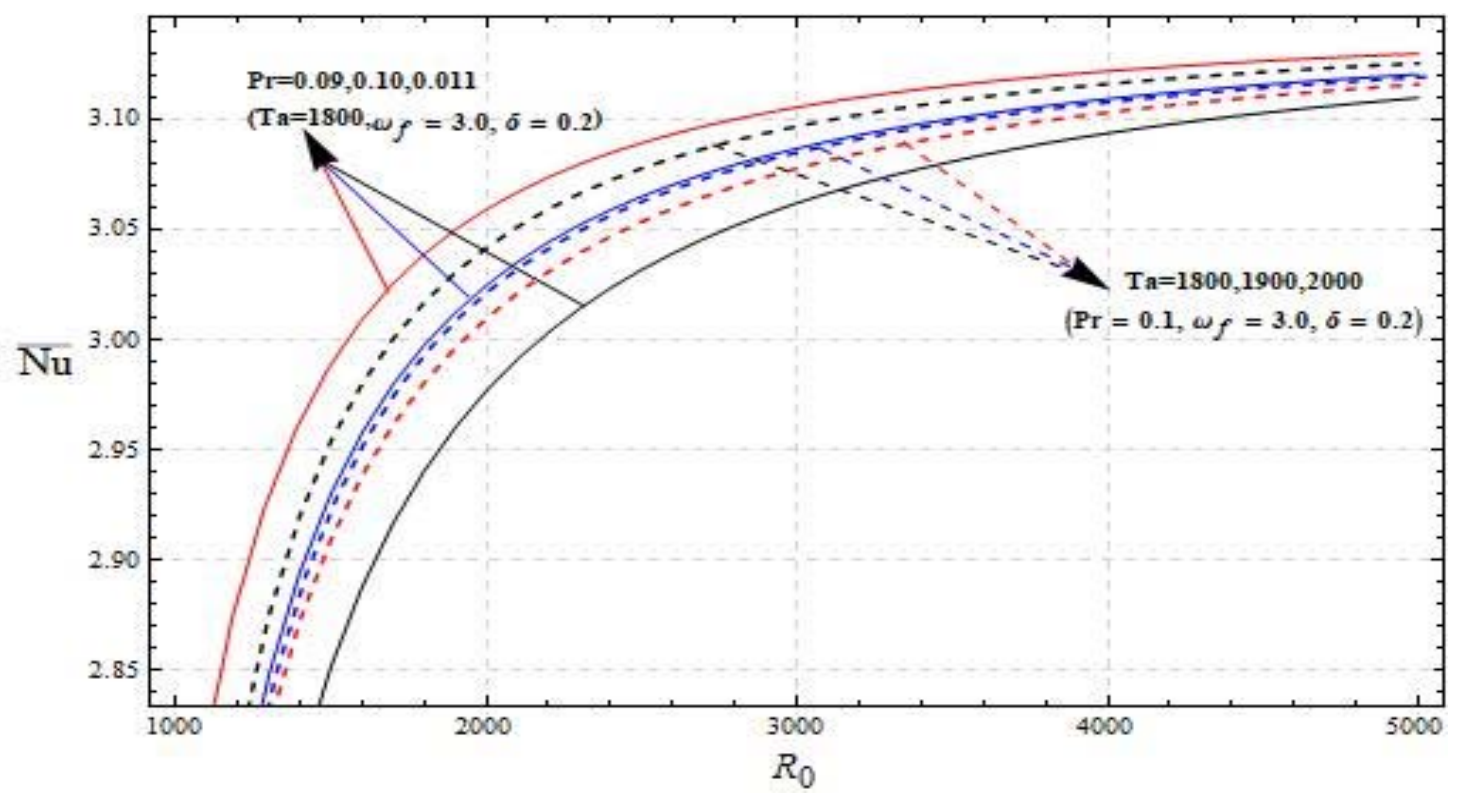

Fig.10. The effect of Pr and Ta on mean Nusselt number Nu.

The mean Nusselt number $(\mathrm{Nu})$ is derived as a function of $R O$ from Eq.(3.21) and the corresponding results are presented in Fig.10. A similar effect of Pr and Ta on the mean Nusselt number is found. They enhance and reduce heat transfer, respectively, and it can be concluded that $\operatorname{Pr}$ destabilizes and Ta stabilizes the system. These results confirm to the results obtained by Kumar and Bhadauria [33].

\section{Conclusions}

The effect of rotation speed modulation is investigated for the oscillatory mode of convection by performing a weakly nonlinear stability analysis resulting in the complex Ginzburg-Landau model. The following conclusions are made from our analysis: 
1. The effect of $\operatorname{Pr}$ is to enhance the heat transfer.

2. The rotation has dual effects either to diminish (for $\mathrm{Ta}<6000$ ) or enhance (for $\mathrm{Ta}>6000$ ) heat transfer.

3. Only for lower values of $\omega f$ heat transfer is maximum.

4. The effect of amplitude $\delta$ of modulation is to enhance heat transfer.

5. Heat transfer is greater for the oscillatory mode of convection than for the stationary mode.

6. Rotation speed modulation reduces heat transfer more than gravity and thermal modulations.

7. The effect of both modulation and rotation disappear for higher values of $\omega f$.

\section{Acknowledgement}

The first author Dr S.H. Manjula is grateful to her Institute VFSTR for providing research specialties in the Department. The author Dr. Palle Kiran is grateful to his Institute CBIT for providing research specialties in the Department. Finally, the authors are grateful to the referees for their most valuable comments that improved the paper considerably. Further the authors RR and BSB acknowledge the support of their institutions.

\section{Nomenclature}

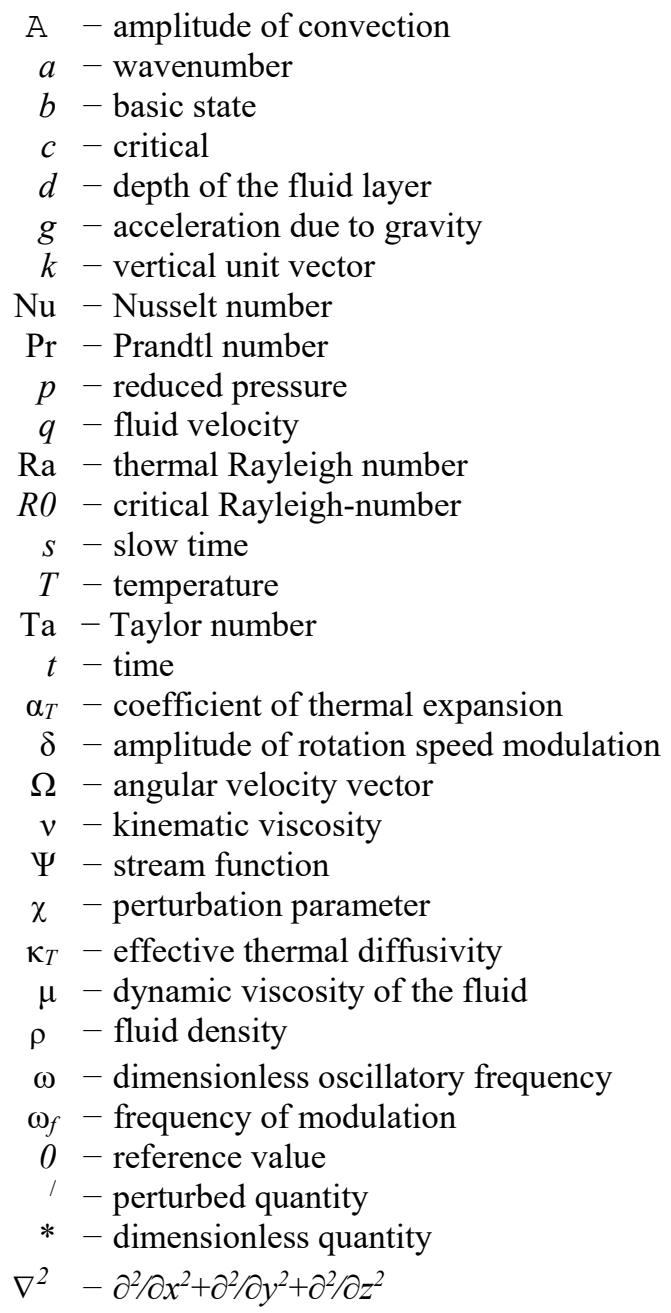




\section{References}

[1] Chandrasekhar S. (1961): Hydrodynamic and Hydromagnetic Stability. - Oxford, UK: Oxford University Press.

[2] Drazin P.G. and Reid D.H. (2004): Hydrodynamic Stability. - Cambridge, UK: Cambridge University Press.

[3] Donnelly R.J. (1964): Experiments on the stability of viscous flow between rotating cylinders III: enhancement of hydrodynamic stability by modulation. - Proc. R. Soc. Lond. Ser A. vol.281, pp.130-139.

[4] Venezian G. (1969): Effect of modulation on the onset of thermal convection. - J. Fluid Mech. vol.35, pp.243-254.

[5] Gresho P.M. and Sani R.L. (1970): The effects of gravity modulation on the stability of a heated fluid layer. - J. Fluid Mech. vol.40, pp.783-806.

[6] Ahlers G., Hohenberg P.C. and Lucke M. (1985): Thermal convection under external modulation of the driving force. - I The Lorenz Model Phys. Rev. A. vol.32, No.6, pp.3493-3518.

[7] Niemela J.J. and Donnelly R.J. (1986): Direct transition to turbulence in rotating Bénard convection. - Phys. Rev. Lett. vol.57, pp.2524-2527.

[8] Walsh T.J. and Donnelly R.J. (1988): Taylor-Couette flow with periodically co-rotated and counters rotated cylinders. - Phys.. Rev. Lett., vol.60, pp.700-703.

[9] Kiran P., Manjula S.H. and Narasimhulu Y. (2018): Weakly nonlinear oscillatory convection in a viscoelastic fluid saturated porous medium with throughflow and temperature modulation. - Int. J. of Applied Mech. and Engg., vol.23, No.3, pp.635-653 https://doi.org/10.2478/ijame-2018-0035

[10] Kiran P. and Narasimhulu Y. (2018): Internal heating and thermal modulation effects on chaotic convection in a porous medium. - J. of Nanofluids, vol.7, pp.544-555.

[11] Manjula S.H., Kiran P. and Narasimhulu Y. (2018): Heat transport in a porous medium saturated with variable viscosity under the effects of thermal modulation and internal heating. - Int. J. of Emerging Tech. and Innovative Res., vol.5, pp.59-75.

[12] Bhattacharjee J.K. (1989): Rotating Rayleigh-Bénard convection with modulation. - J. Phys. A Math. Gen., vol.22, pp.L1135-L1139.

[13] Suthar Om.P., Bhadauria B.S. and Khan A. (2009): Modulated centrifugal convection in a rotating vertical porous layer distant from the axis of rotation. - Transp. Porous Media, vol.79, No.2, pp.255-264.

[14] Suthar Om.P., Bhadauria B.S. and Khan A. (2011): Rotating Brinkman-Lapwood convection with modulation.Transp Porous Media, vol.88, pp.369-383.

[15] Bhadauria B.S and Kiran P. (2014): Effect of rotational speed modulation on heat transport in a fluid layer with temperature dependent viscosity and internal heat source. - Ain Shams Engineering J., vol.5, pp.1287-1297.

[16] Kiran P., Manjula S.H. and Narasimhulu Y. (2018): Oscillatory convection in a rotating fluid layer under gravity modulation. - J. of Emerging Tech. and Innovative Res., vol.5, No., pp.227-242.

[17] Rauscher J.W. and Kelly R.E. (1975): Effect of modulation on the onset of thermal convection in a rotating fluid. Int. J. Heat Mass Transfer, vol.18, pp.1216-1217.

[18] Malashetty M.S. and Swamy M. (2008): Effect of thermal modulation on the onset of convection in a rotating fluid layer. - Int. J. Heat Mass Transfer, vol.51, pp.2814-2823.

[19] Bhadauria B.S., Siddheshwar P.G. and Suthar OM.P. (2012): Nonlinear thermal instability in a rotating viscous fluid layer under temperature/gravity modulation. - J. Heat Transfer, vol.134, No.10, pp.102502.

[20] Bhadauria B.S and Kiran P. (2014): Weak nonlinear oscillatory convection in a viscoelastic fluid layer under gravity modulation. - Int. J. Nonlinear Mech., vol.65, pp.133-140.

[21] Bhadauria B.S. and Kiran P .(2014): Weak nonlinear oscillatory convection in a viscoelastic fluid saturated porous medium under gravity modulation. - Transp. Porous Media, vol.104, pp.451-467. 
[22] Kiran P. and Bhadauria B.S. (2016): Weakly nonlinear oscillatory convection in a rotating fluid layer under temperature modulation. - J. of Heat Transf., vol.138, pp.051702.

[23] Kiran P., Bhadauria B.S. and Narasimhulu Y. (2016): Nonlinear throughflow effects on thermally modulated rotating porous medium. - J. of Applied Nonlinear Dynamics, vol.6.

[24] Kiran P. (2016): Nonlinear throughflow and internal heating effects on vibrating porous medium. - Alexandria Engineering J., vol.55, pp.757-767. doi.org/10.1016/j.aej.2016.01.012

[25] Kiran P. and Narasimhulu Y. (2017): Centrifugally driven convection in a nanofluid saturated rotating porous medium with modulation. - J. of Nanofluid, vol.6, No.1, pp.01-11.

[26] Kiran P., Bhadauria B.S. and Narasimhulu Y. (2018): Oscillatory magneto-convection under magnetic field modulation. - Alexandria Engg. J., vol.57, pp.445-453.

[27] Kim M.C., Lee S.B., Kim S. and Chung B.J. (2003): Thermal instability of viscoelastic fluids in porous media. - Int. J. Heat Mass Transf., vol.46, pp.5065-5072.

[28] Bhadauria B.S. and Kiran P. (2014): Weakly nonlinear oscillatory convection in a viscoelastic fluid saturating porous medium under temperature modulation. - Int. J. Heat Mass Transfer, vol.77, pp.843-851.

[29] Bhadauria B.S. and Kiran P. (2014): Heat and mass transfer for oscillatory convection in a binary viscoelastic fluid layer subjected to temperature modulation at the boundaries. - Int. Commun. in Heat and Mass Transf., vol.58, pp.166-175.

[30] Malkus W.V.R. and Veronis G. (1958): Finite amplitude cellular convection. - J. Fluid Mech., vol.4, No.3, pp.225260.

[31] Bhadauria B.S. and Kiran P. (2014): Weak nonlinear analysis of magneto-convection under magnetic field modulation. - Physica Scripta, vol.89, No.9, pp.095209.

[32] Vadász P. (1998): Coriolis effect on gravity-driven convection in a rotating porous layer heated from below. - J. Fluid Mech., vol.376, pp.351-375.

[33] Kumar A. and Bhadauria B.S. (2011): Thermal instability in a rotating anisotropic porous layer saturated by a viscoelastic fluid. - Int. J. Non-Linear Mech., vol.46, pp.47-56.

[34] Veronis G. (1958): Cellular convection with finite amplitude in a rotating fluid. - J.. Fluid Mech., vol.5, pp.401-435.

[35] Siddheshwar P.G., Bhadauria B.S., Mishra P. and Srivastava A.K. (2012): Study of heat transport by stationary magneto-convection in a Newtonian liquid under temperature or gravity modulation using Ginzburg-Landau model. - Int. J. of Non-Linear Mechanics, vol.47, pp.418-425.

[36] Bhadauria B.S. and Kiran P. (2015): Chaotic and oscillatory magneto-convection in a binary viscoelastic fluid under G-jitter. - Int. J. Heat Mass Transf., vol.84, pp.610-624.

[37] Kiran P. (2015): Throughow and G-jitter effects on binary fluid saturated porous medium. - Applied Math. and Mech., vol.36, No.10, pp.1285-1304.

[38] Kiran P and Bhadauria B.S. (2015): Chaotic convection in a porous medium under temperature modulation. Transp. Porous Media, vol.107, pp.74-763.

[39] Bhadauria B.S., Singh M.K., Singh A., Singh B.K. and Kiran P. (2016): Stability analysis and internal heating effects on oscillatory convection in a viscoelastic fluid saturated porous medium under gravitation modulations. - Int. J. Appl. Mech. Engng., vol.21, pp.785-803.

[40] Kiran P. (2019): Concentration modulation effect on weakly nonlinear thermal instability in a rotating porous medium. - J. of Applied Fluid Mechanics. $2^{\text {nd }}$ revised. 